\title{
Clinical and Surgical Outcome of a Supplementary Multifocal Intraocular Lens Implanted with a Bag-In-the-Lens Intraocular Lens: 5-Year Follow-Up
}

\author{
Thomas Verdonck ${ }^{a, b}$ Liliana Werner ${ }^{c}$ Sorcha Ní Dhubhghaill ${ }^{a, b}$ \\ Marie-José Tassignon ${ }^{a, b}$

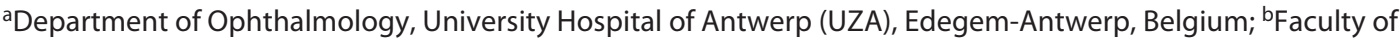 \\ Medicine and Health Sciences, University of Antwerp (UAntwerpen), Antwerp, Belgium; 'John A. Moran Eye Center, \\ Salt Lake City, UT, USA
}

\section{Keywords}

Supplementary intraocular lens $\cdot$ implantation · Rayner sulcoflex · Dysphotopsia · Pigment deposits · Presbyopia correction

\begin{abstract}
Introduction: To assess the postoperative outcome and patient-reported satisfaction, spectacle independence, and dysphotopsia after implantation with the mutifocal Rayner Sulcoflex supplementary intraocular lens (sIOL). Materials and Methods: We analyzed the outcome of all patients implanted with a multifocal sIOL between 2009 and 2011. In all cases, the sIOL was the Rayner Sulcoflex IOL (type 653F) and the primary IOL was a bag-in-the-lens (BIL) IOL. The data were obtained through a retrospective analysis of the patient records and by means of a questionnaire, 5 years after sIOL implantation. Results: This study included 31 eyes of 20 patients. In 13 eyes, the sIOL needed explantation $(n=13$, 41.94\%). Dysphotopsia is a frequent complaint (12/15 patients: $80.0 \%$ ) after sIOL implantation. In 13 out of 31 eyes (41.94\%), pigment deposits were found on the sIOL with variable clinical complaints. Five eyes required additional surgery because of clinically significant deposits. Discus-
\end{abstract}

sion/Conclusion: Patients with a multifocal sIOL in combination with a BIL implantation have a higher incidence of dysphotopsia compared to previously published studies. Dysphotopsia was the main complaint and reason for explantation. We encountered a high incidence of pigmented IOL deposits. The sIOLs can be safely removed even years after implantation.

c) 2020 S. Karger AG, Basel

\section{Introduction}

Monofocal intraocular lenses (IOLs) provide an excellent means through which vision can be restored for a chosen target refraction following cataract or clear lens extraction. One significant limitation of the monofocal approach is that they are usually accompanied by spectacle dependence for other focal points, unless monovision is applied. The "pseudophakic presbyopia," induced by monofocal implants, is due to their inability to replicate the accommodation seen in the natural crystalline lens. Discovering the perfect lens implant, which would correct presbyopia and provide true spectacle independence, remains a major goal of research. Numerous surgical de- karger@karger.com

www.karger.com/ore

(C) 2020 S. Karger AG, Base

Karger"
Thomas Verdonck

Department of Ophtalmology, University Hospital of Antwerp Wilrijkstraat 10

BE-2650 Edegem (Belgium)

thomasverdonck@telenet.be 
Table 1. Patient questionnaire (also available in Dutch and French) and results

General

1. Was the lens explanted in a different center?

2. Did you undergo any other surgeries on your eye(s)?
(Yes/no)

(Yes/no; if yes specify)

(Weak/reasonable/good/very good/excellent)

(Not at all/barely/considerably/a lot/made impossible)

Watching television?

Driving during daytime?

Driving during night-time?

Recognizing faces on the street?

Reading price tags at the store?

Doing fine work, like knitting?

Dysphotopsia

5. How much are you limited by dysphotopsia in each of the following activities:

Your daily activities?

Reading reflective paper?

(Not at all/barely/considerably/a lot/made impossible)

Driving a car?

Walking outside?

Satisfaction

6. How satisfied are you about your:

Distance vision?

Intermediate vision?

Near vision?

7. Would you have the same lens implanted again?

8. Would you recommend this lens to a family member or a relative?

Spectacle independence

9. Do you wear spectacles or contact lenses during much of the day?

10. Do you wear spectacles or contact lenses during one of these activities?

Daily activities?

Reading price tags?

Reading a book/magazine?
(Very unsatisfied/unsatisfied/ neutral/satisfied/very

satisfied)

(Definitely not/probably not/neutral/probably/definitely)

(Definitely not/probably not/neutral/probably/definitely)

Questionnaire results - respondents, $n=15$ (75\%)

\begin{tabular}{|c|c|c|}
\hline Respondents results & $n$ & $\%$ \\
\hline Explanted & 7 & 47 \\
\hline Satisfaction & 8 & 53 \\
\hline Dysphotopsia & 12 & 80 \\
\hline Mild & 6 & 40 \\
\hline Moderate & 4 & 27 \\
\hline Severe & 2 & 14 \\
\hline Spectacle independence (unexplanted) & 6 & 100 \\
\hline
\end{tabular}

vices and approaches are currently being explored with this goal in mind. The use of multifocal IOLs is increasing the last decade among surgeons seeking to offer their patients a solution to presbyopia. There remains however a significant number of patients who are dissatisfied with the postoperative outcomes and appear to be intolerant for multifocal optics. The most frequent problems reported by patients after multifocal IOLs are as follows: (1) blurred vision due to ametropia, dry eye syndrome, posterior capsule opacification, or a large pupil and (2) dysphotopsia due to IOL decentration. Multifocal lens dissatisfaction is an increasingly common indication for lens explantation. However, these surgeries cannot be pro-
(Yes/no)

(Yes/no)

Watching television?

Driving a car? 
Table 2. Patient and eye characteristics

Patient characteristics $(n=20)$

Bilateral

Sex

Age, yr

Eye characteristics $(n=31)$

Side

Comorbidities

Simultaneous implantation

Interval, $\mathrm{m}$

Anesthesia

\author{
Yes $(n=11 ; 55.00 \%)$ \\ Female $(n=10 ; 50.00 \%)$ \\ $55.60 \pm 13.96$ [24-77]
}

$$
\begin{aligned}
& \text { No }(n=9 ; 45.00 \%) \\
& \text { Male }(n=10 ; 50.00 \%)
\end{aligned}
$$

\author{
$\operatorname{RE}(n=15 ; 48.39 \%)$ \\ Retinal problems $(n=2 ; 6.45 \%)$ \\ Yes $(n=21 ; 67.74 \%)$ \\ $28.88 \pm 25.84[5-70]$ \\ Topical $(n=24 ; 77.42 \%)$
}

\author{
LE $(n=16 ; 51.61 \%)$ \\ Corneal problems $(n=1 ; 3.23 \%)$ \\ No $(n=10 ; 53 \%)$
}

Retrobulbar $(n=1 ; 3.23 \%)$

General $(n=6 ; 19.35 \%)$

tifocal version as an alternative to multifocal IOL implantation $[6,7]$. The idea was that in case of patient dissatisfaction, the supplementary IOL could be exchanged very easily. The aim of this study was to examine the 5-year follow-up data of a cohort of patients who had a cataract surgery with primary monofocal IOL implantation and subsequent implantation of a Rayner Sulcoflex multifocal sIOL in the same or different surgical procedure.

\section{Patients and Methods}

In this retrospective study, we analyzed the outcome for all patients who received a multifocal Rayner Sulcoflex sIOL following the protocol approved by the Ethics Committee of the Antwerp University (EC protocol 9/39/189). The sIOLs were provided free by the company for the study patients.

All implantations occurred between 2009 and 2011. The data were obtained through a retrospective analysis of the patient records 5 years after follow-up and by means of a questionnaire (Table 1). The questionnaire was based on a protocol approved by the Hospital Ethics Committee (EC protocol 16/45/476).

\section{Preoperative Assessment}

All patients underwent a preoperative intake consultation including objective and subjective refraction, visual acuity (best-corrected visual acuity and uncorrected visual acuity) both for near and distance vision, slit lamp examination, intraocular pressure, and biometry: axial length, anterior chamber depth, and keratometry.

\section{Intraocular Lenses}

The Rayner Sulcoflex IOL is a 1-piece hydrophilic acrylic lens with undulating $\mathrm{C}$ loop haptics and a posterior haptic vaulting of $10^{\circ}$. The optic diameter is $6.50 \mathrm{~mm}$, and overall length is $14.0 \mathrm{~mm}$. Its optic has a convex-concave shape. The multifocal optic has an addition of $+3.50 \mathrm{D}$ (the equivalent of $\mathrm{a}+3.0 \mathrm{D}$ addition at the spectacle plane).

The primary IOL was a bag-in-the-lens (BIL) Morcher 89A IOL for all cases. The BIL IOL is a hydrophilic acrylic monofocal IOL with a unique design and implantation technique [8]. It was developed to prevent posterior capsule opacification and subse-

Clinical and Surgical Outcome of a

Supplementary Multifocal IOL quent YAG laser capsulotomy. It has a $5.0 \mathrm{~mm}$ central optic, which is surrounded by 2 flanges defining a groove allowing suspension of the IOL by both the anterior and posterior capsulorhexes. In 2 eyes, the BIL IOL was toric. All lens surgeries were performed by a single surgeon (M.J.T.).

\section{Postoperative Assessment}

Postoperative examinations were performed at 1 week and 1 month. The parameters collected were corrected and uncorrected VA for far and near distance, refraction, intraocular pressure, and eventual complications. Subjective assessment of the position of both IOLs and the grade of inflammation and pigment dispersion was made on slit lamp examination. The ray tracing device at our disposal in that time did not allow for evaluation of internal aberration. This was followed by a retinal examination.

Long-term follow-up was performed by a questionnaire (Table 1). We aimed to analyze the following parameters: patient satisfaction, dysphotopsia, spectacle independence, and IOL explantation. This questionnaire was only used for descriptive statistics.

\section{Results}

\section{Demographics}

In total, 31 eyes of 20 patients were included in the data analysis (Table 2). No cases operated during that time were excluded. The cohort consisted of $50 \%$ female and $50 \%$ male with a mean age of 55.60 years \pm 13.96 (SD) (range 24-77 years).

The implantation of the primary IOL and the SIOL occurred simultaneously in 21 eyes (67.74\%). In 4/21 eyes, the primary IOL was exchanged first for a BIL and a sIOL was then implanted consequently in the same surgical procedure. IOL exchange was performed due to the following: (1) multifocal IOL decentration of primary implanted IOL, (2) intolerance of monovision, and (3) refractive surprise after primary surgery. The rationale to combine IOL exchange and sIOL was to solve the case in 1 and the same surgical procedure. 
Table 3. Description of 9 patients with IOL explantation

\begin{tabular}{lllllc}
\hline Patient & Bilateral & Dysphotopsia & IOL deposits & Primary IOL & $\begin{array}{l}\text { Interval, } \\
\text { m RE/LE }\end{array}$ \\
\hline 1 & & & & & Morcher BIL 89A \\
2 & No & No & Yes & Morcher BIL 89A Toric & $2 / 2$ \\
3 & Yes & Yes & Yes & Morcher BIL 89A & $2 / 3$ \\
4 & Yes & Yes & No & Morcher BIL 89A & $-/ 2$ \\
5 & No & Yes & No & Morcher BIL 89A & $-/ 9$ \\
6 & No & Yes & No & Morcher BIL 89A & $5 / 5$ \\
7 & Yes & Yes & No & Morcher BIL 89A & $-/ 1$ \\
8 & No & Yes & No & Morcher BIL 89A & $1 / 4$ \\
9 & Yes & Yes & No & Morcher BIL 89A & $11 / 11$ \\
\hline
\end{tabular}

IOL, intraocular lens; BIL, bag-in-the-lens.

In the remaining 10 eyes (32.26\%), implantation of the sIOLs occurred in 2 different surgical steps with a mean interval between both surgeries of 28.88 months \pm 25.84 (SD) (range 5-8 months). In 3 eyes, the primary IOL was a toric BIL.

In 24 eyes of 16 patients ( $81.08 \%$ ), the surgery was performed under topical and intracameral anesthesia, 3 patients had bilateral surgery (16.22\%) under general anesthesia, and 1 eye of 1 patient was performed under retrobulbar anesthesia $(2.70 \%)$ because of the combination of vitrectomy, cataract, and sIOL implantation. Both the primary and the sIOL implantation were reported as uneventful surgeries in all 31 eyes.

The indications for unilateral surgery were as follows: (1) unilateral cataract, (2) IOL decentration with dysphotopsia in 1 eye of patients implanted with multifocal IOLs in both eyes and satisfied with their contralateral eye, (3) suppressed amblyopic contralateral eye, (4) intolerance of a previously induced monovision, (5) unsatisfied patients after operation of the first eye and who withdrew the surgery of the second eye, and (6) patients who were already operated in 1 eye with a multifocal IOL.

\section{Preoperative Comorbidities}

One patient had a macular scar preoperatively, due to central serous retinopathy 10 years previously, and had normal visual acuity and macula in the other eye. This was the only patient presenting a retinal comorbidity.

\section{Postoperative Visual and Refractive Outcomes}

The mean preoperative CDVA available of 28 eyes was $0.86 \pm 0.27$ (SD) (range 0.05-1.25), and the mean postoperative CDVA visual acuity after 1 month available of 31 eyes was $0.86 \pm 0.27$ (SD) (range 0.05-1.25). The UDVA was $0.81 \pm 0.28$ (range $0.05-1.25$ ) after 1-month followup for the same group of eyes. After 1 month, the total mean postoperative spherical refraction was $0.08 \pm 0.42$ $\mathrm{D}$ (range -1 to +0.75 ) and the total mean cylindrical refraction was $-0.38 \pm 0.73 \mathrm{D}$ (range -2.5 to 0 ).

\section{IOL Explantation}

The total number of sIOL explantations (Table 3) was 13 eyes $(41.93 \%)$ of 8 patients $(40.0 \%)$. Early explantation $(<1$ year postoperatively) occurred in 12 eyes $(38.71 \%)$ of 9 patients (45.0\%) because of unacceptable subjective dysphotopsia. The mean interval before explantation was $3.92 \pm 3.18$ months (SD) (range 1.00-11.00 months). All patients were subjectively relieved after explantation except 1 whose visual complaints persisted. One patient underwent late explantation because of pigment deposits on the sIOL. In this case, the time interval before explantation was 74 months. The mean follow-up of the eyes without explantation ( $n=18$ eyes; $58.06 \%$ ) was 35.39 months \pm 29.12 (SD) (range 0.00-84.00).

\section{IOL Deposits}

There were 13 eyes (41.94\%) of 8 patients in our series in which deposits were found on the sIOL with variable clinical complaints of which the detailed description is summarized in Table 4 . Three eyes of 2 patients (patient 1 and 3) underwent explantation of the sIOL based on the severity of their subjective complaints and impossibility of surgical cleaning of the sIOLs.

In the first explantation case (Fig. 1,2), the patient was planned for sIOL surface cleaning. Preoperatively, it became obvious that the deposits on the sIOL were impossible to clean, justifying the subsequent sIOL removal. Even after explantation, it was not possible to remove the 
Table 4. Description of 8 patients with IOL deposits

\begin{tabular}{|c|c|c|c|c|}
\hline \multirow[t]{2}{*}{$N=13$} & \multirow[t]{2}{*}{ Clinical appearance } & \multirow[t]{2}{*}{ Therapy } & \multicolumn{2}{|l|}{ Interval, $\mathrm{m}$} \\
\hline & & & diagnosis & surgery \\
\hline Patient 1 (unilateral) & $\begin{array}{l}\text { Extensive protein deposits on anterior sIOL surface } \\
\text { and in the IL interface }\end{array}$ & $\begin{array}{l}\text { Cleaning sIOL surface was unsuccessful, } \\
\text { so the sIOL was removed }\end{array}$ & RE: 71 & RE: 74 \\
\hline Patient 2 (unilateral) & Moderate pigment deposits on anterior sIOL surface & $\begin{array}{l}\text { Bilateral IOL removal because of combined } \\
\text { bad image quality }\end{array}$ & RE + LE: 1 & RE + LE: 2 \\
\hline Patient 3 (bilateral) & $\begin{array}{l}\text { Pigment membrane on the anterior sIOL surface and } \\
\text { in the IL-interface }\end{array}$ & $\begin{array}{l}\text { Bilateral peeling and cleaning of pigment } \\
\text { membrane. No removal }\end{array}$ & RE + LE: 82 & $\begin{array}{l}\text { RE: } 83 \\
\text { LE: } 84\end{array}$ \\
\hline Patient 4 (bilateral) & Minor pigment deposits on anterior sIOL surface & Bilateral Nd:YAG + PI & $\mathrm{RE}+\mathrm{LE}: 13$ & RE + LE: 30 \\
\hline Patient 5 (unilateral) & Minor pigment deposits on anterior sIOL surface & No intervention & RE: 7 & na \\
\hline Patient 6 (bilateral) & Minor pigment deposits on anterior sIOL surface & No intervention & $\mathrm{RE}+\mathrm{LE}: 12$ & na \\
\hline Patient 7 (bilateral) & Minor pigment deposits on anterior sIOL surface & No intervention & $\mathrm{RE}+\mathrm{LE}: 18$ & na \\
\hline Patient 8 (unilateral) & Minor pigment deposits on anterior sIOL surface & No intervention & RE: 1 & na \\
\hline
\end{tabular}

IL, interlenticular; IOL, intraocular lens; sIOL, supplementary intraocular lens; PI, peripheral iridotomy, Nd:YAG, neodymium-doped yttrium aluminum garnet.

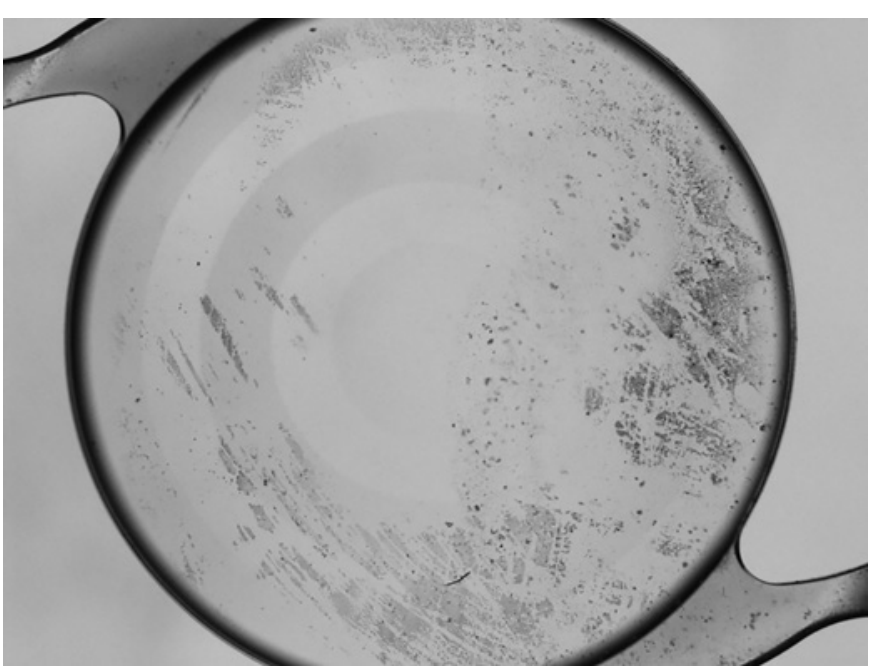

Fig. 1. Protein deposits on explanted Rayner Sulcoflex sIOL (patient 1 , sIOL after explantation; white background). sIOL, supplementary intraocular lens.

deposits of the sIOL using a soft brush. The IOL was sent to the John A. Moran Eye Center for further examination in the hope to discover the possible cause and composition of these deposits. They were organized in a round/ linear configuration on the anterior surface of the sIOL and had the appearance of protein deposits. Energy-dispersive X-ray spectroscopy confirmed that they were not calcium phosphate deposits. The patient did not wish to

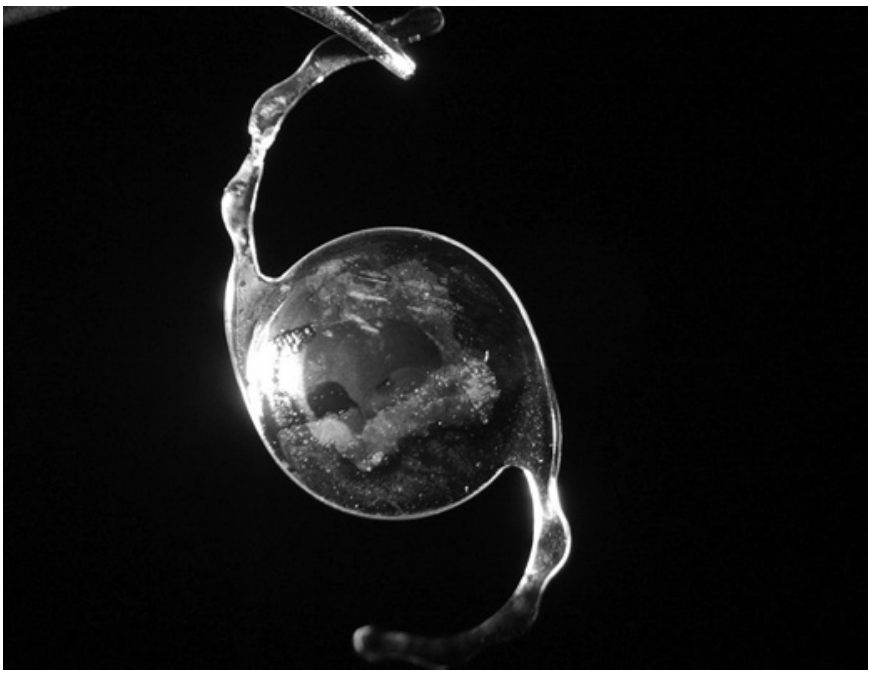

Fig. 2. Protein deposits on explanted Rayner Sulcoflex sIOL (patient 1 , sIOL after explantation; black background). sIOL, supplementary intraocular lens.

be re-implanted with a sIOL and preferred to settle with reading glasses.

In the second explantation case (patient 2), sIOLs showed mild deposits (images not available) and were removed from both eyes on patients' request because of subjective bad quality of vision. The anterior lens deposits in both eyes were clinically compatible with pigment dispersion. The diagnosis was supported by pigment in the anterior chamber 


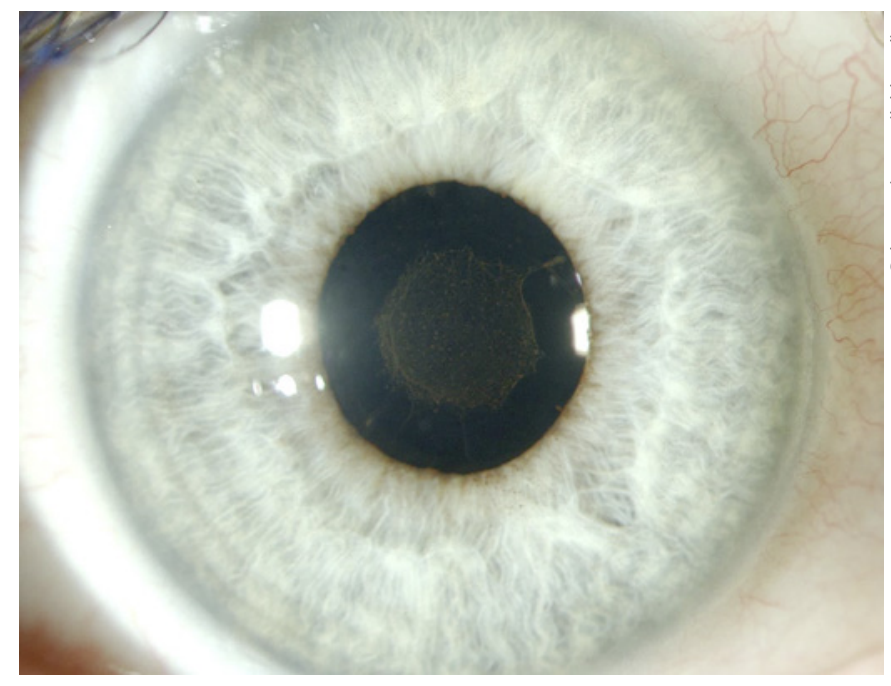

Fig. 3. Pigmented membrane on anterior surface of Rayner Sulcoflex sIOL. (patient 2; right eye, clinical image with normal pupil). sIOL, supplementary intraocular lens.

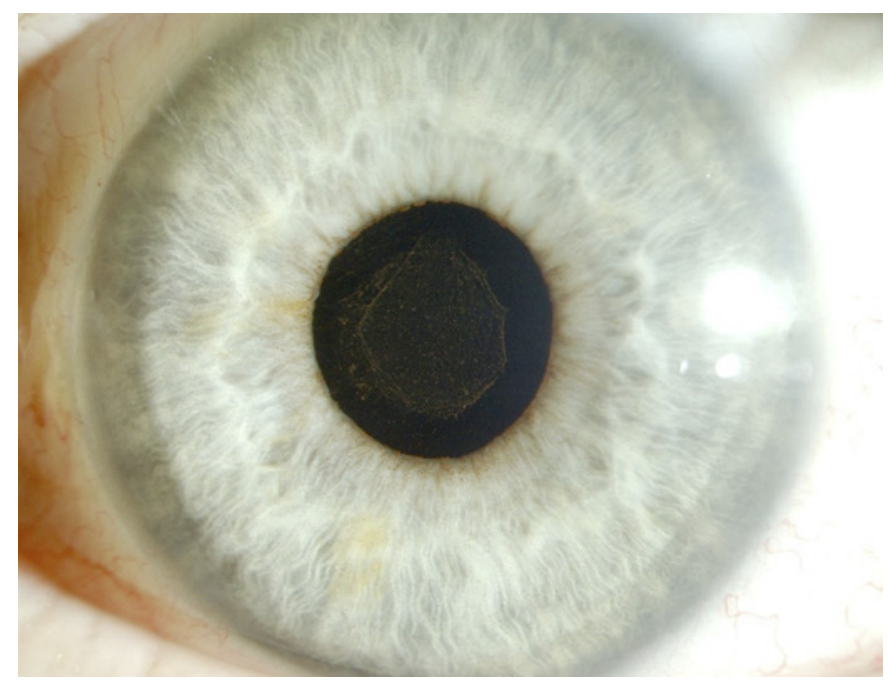

Fig. 5. Pigmented membrane on anterior surface of Rayner Sulcoflex sIOL. (patient 2; left eye, clinical image with normal pupil). sIOL, supplementary intraocular lens.

angle on gonioscopy. On UBM examination, an area of contact between the sIOL and the iris was observed.

In patient 3 (Fig. 3-6), both eyes developed pigmented membranes on the anterior surface of the sIOL as well as interlenticularly. The anterior membrane could be peeled off with a capsulotomy forceps, and the interlenticular membrane aspirated. The postoperative visual acuity was restored, and the patients' complaints resolved.

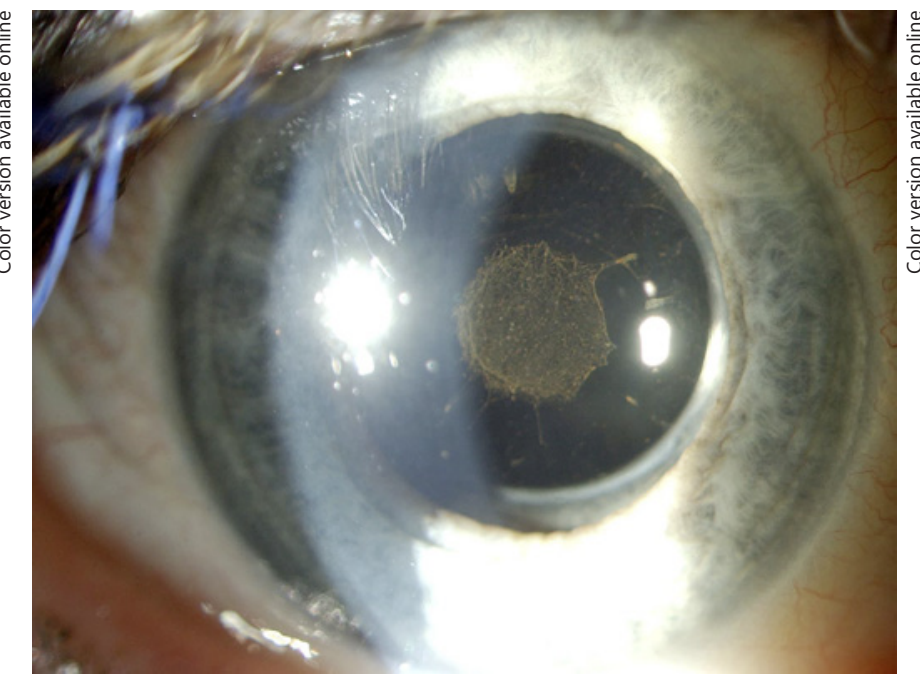

Fig. 4. Pigmented membrane on anterior surface of Rayner Sulcoflex sIOL. (patient 2; right eye, clinical image with mydriatic pupil). sIOL, supplementary intraocular lens.

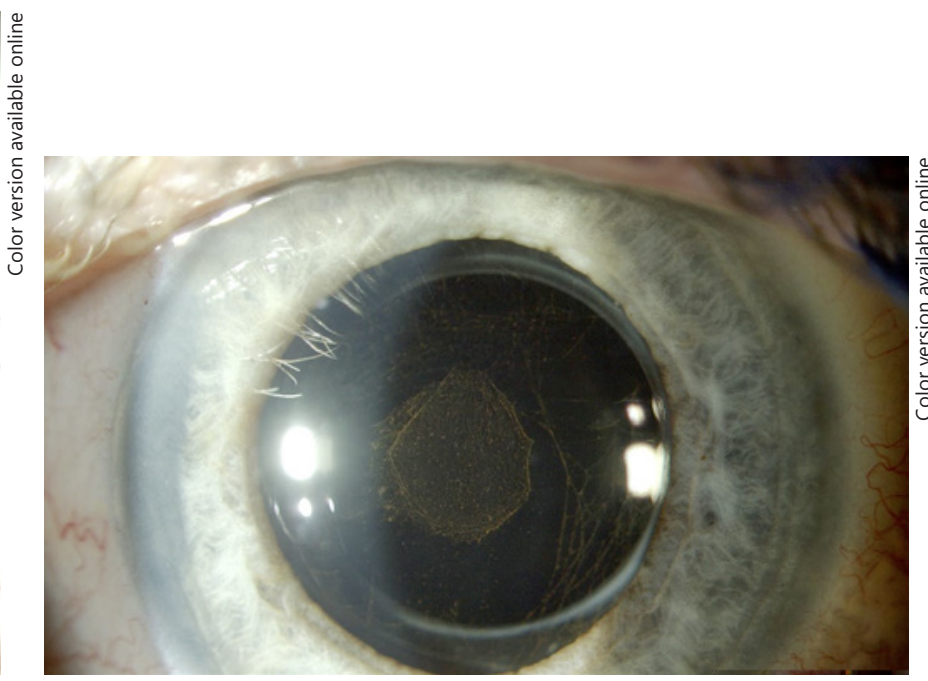

Fig. 6. Pigmented membrane on anterior surface of Rayner Sulcoflex sIOL. (patient 2; left eye, clinical image with mydriatic pupil). sIOL, supplementary intraocular lens.

The other 5 patients (patient 4-8) did not have any subjective complaints caused by the deposits and were satisfied with their lenses. One of these 5 patients had a peripheral YAG iridotomy to prevent pigmentary glaucoma. The patients did not develop any signs of glaucoma during the follow-up period. 
Glaucoma Complications

One patient, who had a sIOL implanted in both eyes, developed open-angle glaucoma in 1 eye. During gonioscopy examination, the anterior chamber angle appeared more pigmented. There were no pigment deposits found on the sIOL and no Kruckenberg's spindle. The glaucoma required medical treatment and laser trabeculoplasty. A second patient, with bilateral sIOL implantation, developed open-angle glaucoma in both eyes, but it could be managed with topical medication.

\section{Retinal Complications}

One eye developed transient cystoid macula edema after a combined vitrectomy (for a macular pucker) and implantation of a BIL IOL with a sIOL. Visual outcome reached 1.0 unaided.

A second patient developed bilateral central serous chorioretinopathy 2 years after the sIOL implantation. After spontaneous regression of the subretinal fluid, the visual acuity recovered to 1.0 in both eyes.

Patient Satisfaction, Spectacle Independence, and Dysphotopsia Complaints (Table 1)

The response rate to the questionnaire sent to all patients of this cohort, independently whether they had sIOL explantation or not, was 15 out of 20 patients ( 6 explanted; 9 not explanted). Based on the questions asked regarding patient satisfaction, $53.3 \%(n=8)$ were satisfied about their sIOL implantation (1 explanted because of IOL deposits; 7 not explanted). Regarding spectacle independency, all patients were spectacle independent for far and intermediate distances. The 6 remaining patients with multifocal sIOL were all satisfied with their near vision.

Dysphotopsia was reported in $80.0 \%$ of respondents $(n=12)$. Patients estimated their dysphotopsia as mild $(n=6)$, moderate $(n=4)$, to severe $(n=2)$.

\section{Discussion}

The latest results of modern multifocal IOLs improved dramatically because better care is taken to patient selection, patient education, and weighing multifocal IOL benefits and side effects. However, there is still a considerable percentage of patients that are not satisfied or are feeling miserable after multifocal IOL implants. These patients will occasionally seek for surgeons willing to perform an IOL exchange.

An advantage of the supplementary IOLs (also known as secondary or additional IOLs) is their position in the sulcus in contrast to the classic piggyback technique, which requires implantation of both the primary and secondary IOLs into the capsular bag. The classic piggyback technique was associated with a number of complications, namely, interlenticular opacification (ILO) [9-12], postoperative elevation of intraocular pressure, pupillary optic capture after mydriasis $[13,14]$, iris chafing, pigment dispersion syndrome $[15,16]$, and secondary pigmentary glaucoma $[15,16]$. The implantation of these IOLs in the ciliary sulcus increases the distance between the 2 IOLs significantly, thereby reducing (but not eradicating) lens-lens contact and the possibility of ILO.

At the time of writing, there were 3 supplementary IOLs, all 3 specifically designed for secondary implantation in the ciliary sulcus to correct corneal astigmatism at the lenticular plane, pseudophakic ametropias, or pseudophakic presbyopia: the Sulcoflex (Rayner Intraocular Lenses Ltd., East Sussex, United Kingdom), which was the first to be made commercially available, the Add-on (HumanOptics, Erlangen, Germany), and A4 AddOn IOL (Medicontur Medical Engineering Ltd., Zsámbék, Hungary/1stQ GmbH, Mannheim, Germany). All of the above multifocal sIOL are withdrawn from the market. Only clinical data for the Rayner and HumanOptics lenses were available in the literature. A study was done to assess the performance and stability of the A4 AddOn IOL in cadaver eyes [17]. We further will refer to this category of supplementary IOLs as sIOLs.

The sIOLs main advantages, as described in the literature, can be summarized as follows:

- Reversibility in comparison to laser procedures [18].

- Easy to both implant and explant compared to in-thebag IOL exchange. However, no data has confirmed this yet [19].

- Easy to calculate the IOL power and good predictability of the refractive outcome since calculation is based on the patient's subjective refraction [20].

Based on this study, we can conclude that explantation of a sIOL went perfectly well in all cases. Not only the procedure went uneventfully but also the postoperative complications were very low. When looking at multifocal sIOLs, its reversibility is an important advantage over a multifocal lens-in-the-bag implantation. The potential complications incurred by classic IOL exchange are indeed avoided with the sIOL solution, such as damage to the zonulae, the posterior capsule, or the anterior hyaloid. This study reports 2 reasons for sIOL removal: bad quality of vision and pigment deposits.

However, only $53 \%$ of the patients with multifocal sIOL implantation were satisfied with their visual quality for near. This is a very low number of satisfaction. In this study, 
all the lenses that were implanted were study lenses offered for free to the patient as a reversible multifocal option. Patients were informed that in case of unacceptable quality of vision, the lens could be removed. This situation might have biased the patient's decision, being more critical and more demanding regarding quality of their sight, to requesting early explantation. When looking at the satisfaction results of the diffractive multifocal supplementary IOL of $\mathrm{Hu}$ manOptics AG, satisfaction rates were comparable to the refractive multifocal sIOL of Rayner [19].

Several publications reported the clinical results of the monofocal Rayner Sulcoflex IOL and HumanOptics Add-on IOL for the correction of residual refractive errors or residual astigmatism after cataract surgery or refractive lens exchange. All papers concluded in positive results and high satisfaction rates regarding safety and predictability [21-25].

The Rayner Sulcoflex lens has also been implanted in patients after corneal surgery to improve refractive outcome, especially in patients not suitable for refractive laser correction [26]. To the best of our best knowledge, only 3 papers were published on the clinical results after multifocal Sulcoflex IOL implantation. Schrecker et al. [27] compared the multifocal refractive Sulcoflex sIOL and the diffractive HumanOptics sIOL. Schrecker et al. $[18,28]$ also compared the multifocal refractive Sulcoflex sIOL with a single diffractive multifocal in the bag IOL. The authors reported a high incidence of mainly mild dysphotopsia. In our series, dysphotopsia was considered severe enough to justify exchange of the sIOL in about $50 \%$. Two other publications $[29,30]$ had only a few cases of multifocal Sulcoflex IOL implantation (respectively $n=4$ and $n=1$ ). We can conclude that dysphotopsia is mainly related to the multifocal properties of the sIOL and was found in literature in both refractive and diffractive sIOL cohorts of patients.

Schrecker et al. [27] reported an incidence of mild pigment dispersion syndrome of 2 eyes of 2 patients (5.7\%) in their patient cohort implanted with the HumanOptics Add-on IOL. Kahraman and Amon [21] reported no cases after a 17-month-long follow-up of 12 eyes implanted. In our population, there was an incidence of pigmentary dispersion secondary to sIOL implantation of $12 / 31$ eyes (41.94\%) of 8 patients, varying from mild to severe. We also had 1 secondary pigmentary glaucoma.

Although the Rayner sIOL has been reported to show calcifications [10], this was not the case in our series. The opacification, shown in Figure 1, was due to protein deposits on the surface of the IOL. It is not clear what the nature of the proteins is and whether they are a precursor of IOL calcification.
Sulcus placement of sIOLs seems to reduce the risk of ILO compared to the classic piggyback IOL implantation, but our hypothesis is that more pigment deposits might occur over time. We therefore recommend regular glaucoma screening of the patients implanted with sIOLs.

Evidence of contact between both lenses was not reported in the follow-up data. This is not expected since both surfaces have a different vaulting. Of those cases where anterior segment images were available (most of the exchanged Sulcoflex lenses), there was no evidence of contact between de lenses within the pupillary area. The BIL IOL has a slightly more anterior position in the eye compared to lens-in-the-bag IOLs. The anterior-posterior thickness is $0.12 \mathrm{~mm}$ thicker for the corresponding lens-in-the-bag IOL type made from the same biomaterial and of the same power than the BIL type. These parameters might cause a slightly more anterior positioning of the BIL IOL-sIOL complex and as a result more prone to induce pigment release from the iris/uvea.

Because this is a retrospective study, the long-term spectacle independence could only be determined subjectively through a questionnaire instead of vision testing as normally performed through UCVA at near and far. Since patient-reported spectacle independence is highly influenced by daily activities and specific job requirements, straightforward yes or no answers to our question regarding satisfaction were not often obtained (Table 4). The patients who had no early sIOL explantation were still satisfied of their near vision after 5 years' follow-up.

Aberrometry was not performed routinely preoperatively. This is a drawback of retrospective studies as we have limited data available about the alignment of the IOLs. This limits us in drawing conclusions as to the optical causes of the dysphotopsia.

\section{Statement of Ethics}

The protocol of the initial study was approved by the Ethics Committee of the Antwerp University (EC protocol 9/39/189). The questionnaire was based on a protocol approved by the Hospital Ethics Committee (EC protocol 16/45/476). The data were obtained through a retrospective analysis of the patient records and by means of a questionnaire, 5 years after sIOL implantation.

\section{Conflict of Interest Statement}

Drs. Verdonck, Werner, and Ni Dhubhghaill have no financial or proprietary interest in any material or method mentioned. Dr. Tassignon has an intellectual property in the bag-in-the-lens implant (US patent 6027 531;EU patent 009406794.PCT/120268) li- 
censed to Morcher GmbH, Stuttgart, Germany. Dr. Liliana Werner is supported in part by an unrestricted grant from Research to Prevent Blindness, Inc., New York, NY, USA, to the Department of Ophthalmology and Visual Sciences, University of Utah; Dr. Werner reports grants and personal fees from Alcon and PowerVision and grants from Anew, Advanced Vision Sciences, Clarvista, Hoya, Physiol, Sharklet, Shifamed, Zeiss, and Perfectlens.

\section{Funding Sources}

The supplementary IOLs were provided free by the company for the study patients.

\section{Author Contributions}

Thomas Verdonck: concept \& design, data acquisition, data analysis \& interpretation, drafting manuscript, critical revision of manuscript, and statistical analysis. Liliana Werner: data analysis \& interpretation, critical revision, and final approval. Sorcha $\mathrm{Ni}$ Dhubhghaill: data analysis \& interpretation, drafting manuscript, supervision, critical revision, and final approval. Marie-José Tassignon: concept \& design, data analysis \& interpretation, critical revision of manuscript, supervision, and final approval.

\section{References}

1 de Vries NE, Nuijts RM. Multifocal intraocular lenses in cataract surgery: literature review of benefits and side effects. J Cataract Refract Surg. 2013 Feb;39(2):268-78.

2 Braga-Mele R, Chang D, Dewey S, Foster G, Henderson BA, Hill W, et al. Multifocal intraocular lenses: relative indications and contraindications for implantation. J Cataract Refract Surg. 2014 Feb;40(2):313-22.

3 Charman WN. Developments in the correction of presbyopia II: surgical approaches. Ophthalmic Physiol Opt. 2014 Jul;34(4):397426.

4 de Silva SR, Evans JR, Kirthi V, Ziaei M, Leyland M. Multifocal versus monofocal intraocular lenses after cataract extraction. Cochrane Database Syst Rev. 2016 Dec 12; 12(12):Cd003169

5 Gundersen KG, Makari S, Ostenstad S, Potvin R. Retreatments after multifocal intraocular lens implantation: an analysis. Clin Ophthalmol. 2016;10:365-71.

6 Sauder G. [Secondary toric intraocular lens implantation in pseudophakic eyes. The addon IOL system]. Ophthalmologe. 2007 Dec; 104(12):1041-5.

7 Amon M. Enhancement of refractive results after cataract surgery and IOL-implantation with a supplementary IOL implanted in the ciliary sulcus. Oftalmologia. 2009;53(4):91-5.

8 Tassignon MJ, Gobin L, Mathysen D, Van Looveren J, De Groot V. Clinical outcomes of cataract surgery after bag-in-the-lens intraocular lens implantation following ISO standard 11979-7:2006. J Cataract Refract Surg. 2011 Dec;37(12):2120-9.

9 Gayton JL, Apple DJ, Peng Q, Visessook N, Sanders V, Werner L, et al. Interlenticular opacification: clinicopathological correlation of a complication of posterior chamber piggyback intraocular lenses. J Cataract Refract Surg. 2000 Mar;26(3):330-6.

10 Werner L, Shugar JK, Apple DJ, Pandey SK, Escobar-Gomez M, Visessook N, et al. Opacification of piggyback IOLs associated with an amorphous material attached to interlenticular surfaces. J Cataract Refract Surg. 2000 Nov;26(11):1612-9.
11 Trivedi RH, Izak AM, Werner L, Macky TA, Pandey SK, Apple DJ. Interlenticular opacification of piggyback intraocular lenses. International ophthalmology clinics. Summer. 2001;41(3):47-62.

12 Werner L, Apple DJ, Pandey SK, Solomon $\mathrm{KD}$, Snyder ME, Brint SF, et al. Analysis of elements of interlenticular opacification. Am J Ophthalmol. 2002 Mar;133(3):320-6.

13 Gayton JL, Sanders V, Van Der Karr M. Pupillary capture of the optic in secondary piggyback implantation. J Cataract Refract Surg. 2001 Sep;27(9):1514-5.

14 Garcia-Feijo J, Saenz-Frances F, MartinezDe-La-Casa JM, Mendez-Hernandez C, Fernandez-Vidal A, Elias-de-Tejada M, et al. Angle-closure glaucoma after piggyback intraocular lens implantation. Eur J Ophthalmol. 2008 Sep-Oct;18(5):822-6.

15 Chang SH, Lim G. Secondary pigmentary glaucoma associated with piggyback intraocular lens implantation. J Cataract Refract Surg. 2004 Oct;30(10):2219-22.

16 Iwase T, Tanaka N. Elevated intraocular pressure in secondary piggyback intraocular lens implantation. J Cataract Refract Surg. 2005 Sep;31(9):1821-3.

17 Reiter N, Werner L, Guan J, Li J, Tsaousis KT, Mamalis N, et al. Assessment of a new hydrophilic acrylic supplementary IOL for sulcus fixation in pseudophakic cadaver eyes. Eye. 2017 May;31(5):802-9.

18 Schrecker J, Kroeber S, Eppig T, Langenbucher A. Additional multifocal sulcus-based intraocular lens: alternative to multifocal intraocular lens in the capsular bag. J Cataract Refract Surg. 2013 Apr;39(4):548-55. Epub 13 Feb 8.

19 Liekfeld A, Ehmer A, Schröter U. Visual function and reading speed after bilateral implantation of 2 types of diffractive multifocal intraocular lenses: add-on versus capsular bag design. J Cataract Refract Surg. 2015 Oct; 41(10):2107-14.

20 Habot-Wilner Z, Sachs D, Cahane M, Alhalel A, Desatnik H, Schwalb E, et al. Refractive results with secondary piggyback implantation to correct pseudophakic refractive errors. J Cataract Refract Surg. 2005 Nov;31(11):2101-3.
21 Kahraman G, Amon M. New supplementary intraocular lens for refractive enhancement in pseudophakic patients. J Cataract Refract Surg. 2010 Jul;36(7):1090-4.

22 Khan MI, Muhtaseb M. Performance of the sulcoflex piggyback intraocular lens in pseudophakic patients. J Refract Surg. 2011 Sep; 27(9):693-6.

23 Basarir B, Kaya V, Altan C, Karakus S, Pinarci EY, Demirok A. The use of a supplemental sulcus fixated IOL (HumanOptics Add-On IOL) to correct pseudophakic refractive errors. Eur J Ophthalmol. 2012 Nov-Dec;22(6): 898-903. Epub 2012 Apr 19.

24 Falzon K, Stewart OG. Correction of undesirable pseudophakic refractive error with the sulcoflex intraocular lens. J Refract Surg. 2012 Sep;28(9):614-9.

25 Ferreira TB, Pinheiro J. Clinical results with a supplementary toric intraocular lens for the correction of astigmatism in pseudophakic patients. Eur J Ophthalmol. 2015 Jul-Aug; 25(4):302-8. Epub 2015 Jan 27.

26 Thomas BC, Auffarth GU, Reiter J, Holzer MP, Rabsilber TM. Implantation of threepiece silicone toric additive IOLs in challenging clinical cases with high astigmatism. J Refract Surg. 2013 Mar;29(3):187-93.

27 Schrecker J, Blass S, Langenbucher A. Silicone-diffractive versus acrylic-refractive supplementary iols: visual performance and manual handling. J Refract Surg. 2014 Jan; 30(1):41-8.

28 Schrecker J, Feith A, Langenbucher A. Comparison of additional pseudophakic multifocal lenses and multifocal intraocular lens in the capsular bag. Br J Ophthalmol. 2014 Jul; 98(7):915-9.

29 Gerten G, Kermani O, Schmiedt K, Farvili E, Foerster A, Oberheide U. Dual intraocular lens implantation: monofocal lens in the bag and additional diffractive multifocal lens in the sulcus. J Cataract Refract Surg. 2009 Dec; 35(12):2136-43.

30 Huerva V. Piggyback multifocal IOLs for a hyperopic-presbyopic surprise after cataract surgery in high myopic patients. Cont Lens Anterior Eye. 2014 Feb;37(1):57-9.
Clinical and Surgical Outcome of a Supplementary Multifocal IOL
Ophthalmic Res 2021;64:503-511 DOI: $10.1159 / 000513790$ 